Karen Melissa Garces Porras

Estudo termodinâmico e cinético da adsorção de chumbo e cadmio em ácido húmico extraído a partir de carvão mineral

Dissertação de Mestrado

Dissertação apresentada como requisito parcial para obtenção do título de Mestre pelo Programa de PósGraduação em Engenharia de Materiais e de Processos Químicos e Metalúrgicos da PUC - Rio.

Orientador: Prof. Eduardo Albuquerque Brocchi Co-orientadora: Profa. Deborah Pinheiro Dick 
Karen Melissa Garces Porras

\section{Estudo termodinâmico e cinético da adsorção de chumbo e cadmio em ácido húmico extraído a partir de carvão} mineral

Dissertação apresentada como requisito parcial para obtenção do título de Mestre pelo Programa de PósGraduação em Engenharia de Materiais e de Processos Químicos e Metalúrgicos da PUC - Rio. Aprovada pela Comissão Examinadora abaixo assinada.

Prof. Eduardo Albuquerque Brocchi Orientador Departamento de Engenharia Química e de Materiais PUC - Rio

Profa. Deborah Pinheiro Dick Co-orientadora Departamento de Engenharia de Materiais PUC - Rio

Profa. Sílvia Cristina Alves França Tecnologista Sênior CETEM

Prof. Luís Alberto Cesar Teixeira Departamento de Engenharia Química e de Materiais PUC - Rio

Prof. José Eugenio Leal Coordenador setorial de Pós-graduação do Centro Técnico Científico da PUC-Rio 
Todos os direitos reservados. É proibida a reprodução total ou parcial do trabalho sem autorização da universidade, do autor e do orientador.

\section{Karen Melissa Garces Porras}

Graduou-se em Engenharia Química pela Universidade Nacional San Antônio Abad do Cusco - Perú em 2011.

Ficha Catalográfica

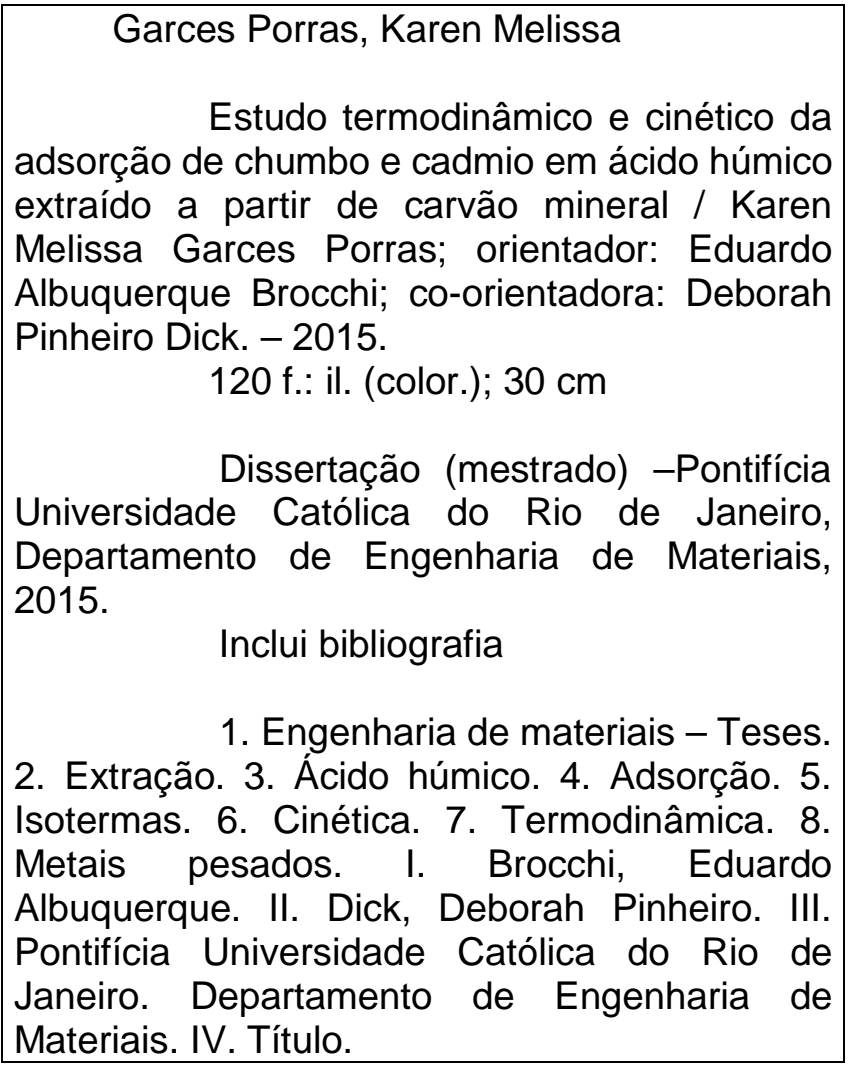

CDD: 620.11 
A meus pais, Manuel e Francisca, por seu amor, trabalho e sacrifício em todos estes anos, por vocês é conseguido chegar até aqui, é um privilégio ser sua filha, vocês são os melhores pais. 


\section{Agradecimentos}

Agradeço a Deus por ter me acompanhado e ser minha fortaleza em tempos de fraqueza.

Agradeço ao meu orientador Dr. Eduardo Albuquerque Brocchi pelo apoio e confiança depositada, e aos professores do Departamento de Engenharia de Materiais da Pontifícia Universidade Católica de Rio de Janeiro - PUC-Rio.

Ao minha co-orientadora Dra. Deborah Pinheiro Dick; pela paciência, ajuda e por ter compartilhado seus conhecimentos em todas as etapas do desenvolvimento de minha dissertação.

Agradeço aos meus pais Manuel e Francisca por todo o seu amor e compreensão ao longo da minha vida. A meus irmãos Elmerth e Fabrízio por me apoiar em tudo momento, e por encher minha vida com grandes momentos de felicidade.

A minha roomie: Deysi por fazer o papel de uma família de verdade. Ao Elvis por ser "ele" quando eu precisei. Aos meus queridos amigos Pamela, Jhonatan, Lili, Paul e Roger pelo carinho demonstrado e fazer a minha vida acadêmica uma jornada de experiências que nunca vou esquecer.

A todos que, direta ou indiretamente, contribuíram para a realização deste trabalho.

Meu agradecimento à Universidade Católica do Rio de Janeiro (PUC), ao CNPQ e FAPERJ, pelo apoio financeiro, durante o curso de mestrado. 


\section{Resumo}

Porras, Karen Melissa Garces; Brocchi, Eduardo Albuquerque. Estudo termodinâmico e cinético da adsorção de chumbo e cadmio em ácido húmico extraído a partir de carvão mineral. Rio de Janeiro, 2015. 120p. Dissertação de mestrado - Departamento de Engenharia de Materiais, Pontifícia Universidade Católica do Rio de Janeiro.

Dentre os contaminantes químicos liberados nos corpos de água, chumbo e cádmio são extremamente prejudiciais para os seres vivos. As sustâncias húmicas, devido à sua estrutura molecular complexa, que compreende grupos carboxílicos, fenólicos e éter, são adsorventes eficazes para poluentes orgânicos e metais. Neste contexto, o principal objetivo do presente trabalho foi avaliar o potencial do ácido húmico $(\mathrm{AH})$ como adsorvente para chumbo e cádmio em meio aquoso e determinar os parâmetros cinéticos e termodinâmicos do processo. AH foi extraído de uma amostra de carvão, coletada na área de mineração do Estado de Santa Catarina, previamente oxidada com $\mathrm{HNO}_{3}$ concentrado em condições de refluxo, conforme a metodologia recomendada pela IHSS. O AH obtido foi caraterizado por técnicas de análise elementar, FTRI, análise termogravimétrica e MEV. Os resultados da caracterização foram similares aos relatados na literatura. Foram realizados ensaios de adsorção para cada cátion, em diferentes níveis de $\mathrm{pH}$ da solução, e a partir desta avaliação previa ficou determinado o valor de $\mathrm{pH}$ em 5. Os ensaios de adsorção com variação da concentração inicial dos cátions foram realizados separadamente para cada metal em batelada, empregando as seguintes condições: $50 \mathrm{~mL}$ de uma solução de cátion ( 0 a $\left.100 \mathrm{mg} \mathrm{L}^{-1}\right), 50 \mathrm{mg}$ de $\mathrm{AH}$, agitação mecânica (175 rpm) e temperatura constante $\left(25^{\circ} \mathrm{C}\right)$. Após 24 horas, os sistemas foram centrifugados e a concentração de cada metal no sobrenadante foi determinada por espectrometria de absorção atómica. As isotermas obtidas para $\mathrm{Pb}$ e $\mathrm{Cd}$ apresentaram uma curva em forma de L, o que indica que os sítios adsorventes disponíveis no $\mathrm{AH}$ tendem à saturação. Comparativamente a capacidade de adsorção máxima para o $\mathrm{Pb}$ foi mais elevada $\left(77 \mathrm{mg} \mathrm{g}^{-1}\right)$ do que aquela apresentada para o Cd $\left(12 \mathrm{mg} \mathrm{g}^{-1}\right)$. Tal fato indica uma maior afinidade dos sítios de adsorção do $\mathrm{AH}$ para o $\mathrm{Pb}$. Comparativamente, foram feitos ensaios de adsorção com AH de Leonardita (padrão da IHSS) e carvão mineral oxidado. Para a determinação dos parâmetros termodinâmicos, foram realizados ensaios de adsorção em três temperaturas diferentes $\left(15,25,35^{\circ} \mathrm{C}\right)$. O 
processo de adsorção foi de natureza endotérmico, com um valor de entalpia de $7,86 \mathrm{~kJ} \mathrm{~mol}^{-1}$ e um valor de entropia de $23,11 \mathrm{~J} \mathrm{~mol}^{-1} \mathrm{~K}^{-1}$. A cinética de adsorção segue um modelo cinético de pseudo-segunda ordem com constates de taxa de $0,065 \mathrm{mg} \mathrm{g}^{-1} \min ^{-1}$ e $0,025 \mathrm{mg} \mathrm{g}^{-1} \min ^{-1}$ para $\mathrm{Pb}\left(\mathrm{C}_{\mathrm{o}} 17,60 \mathrm{mg} \mathrm{L}^{-1}\right)$ e $\mathrm{Cd}\left(\mathrm{C}_{\mathrm{o}} 6,41 \mathrm{mg}\right.$ $\mathrm{L}^{-1}$ ), respetivamente. Os resultados obtidos no presente estudo mostram que o $\mathrm{AH}$ de carvão mineral pode eventualmente ser empregado para a remoção de metais pesados em meio aquoso, porem estudos sobre otimização de seu potencial devem ser todavia realizados.

\section{Palavras chave}

Extração; ácido húmico; adsorção; isotermas; cinética; termodinâmica; metais pesados. 


\section{Abstract}

Porras, Karen Melissa Garces; Brocchi, Eduardo Albuquerque (Advisor). Thermodynamic and Kinetic Studies of the Lead and Cadmium Adsorption in Humic Acid extracted from Coal. Rio de Janeiro, 2015. 120p. Master dissertation - Departamento de Engenharia de Materiais, Pontifícia Universidade Católica do Rio de Janeiro.

Among the released chemical contaminants into water bodies, lead and cadmium are extremely harmful to living things. The humic substances, due to their complex molecular structure that comprises both aliphatic and aromatic moieties as well as carboxylic, phenolic and ether groups are effective adsorbents for organic pollutants and metals. In this context, the main objective of this study was to evaluate the potential of humic acid (HA), extracted from previously oxidized coal, as adsorbent for lead and cadmium in aqueous solutions and determine the kinetic and thermodynamic parameters of the process. HA was extracted from a coal sample, collected in the mining area of the State of Santa Catarina, previously oxidized with $\mathrm{HNO}_{3}$ concentrated in reflux conditions, according to the methodology recommended by the IHSS. The HA obtained was characterized by elemental analysis techniques, FTRI, thermogravimetric analysis and SEM. The characterization results were similar to those reported in the literature. Adsorption experiments were carried out for each cation in different $\mathrm{pH}$ levels, and from this evaluation it was determined the value of $\mathrm{pH}$ at 5 . The adsorption experiments with a variation of the initial concentration of cations were performed separately for each metal by employing the following conditions: $50 \mathrm{ml}$ of a cation solution (0 to 100 mg L-1), $50 \mathrm{mg}$ of HA, mechanical agitation (175 rpm) and constant temperature $\left(25^{\circ} \mathrm{C}\right)$. After 24 hours, the system was centrifuged and the concentration of each metal in the supernatant was determined by atomic absorption spectrometry (AA). The isotherms obtained for $\mathrm{Pb}$ and $\mathrm{Cd}$ showed a L-shaped curve, indicating that the available sorptive sites on HA tending to a saturation point. Compared maximum adsorption capacity for $\mathrm{Pb}$ was higher $\left(77 \mathrm{mg} \mathrm{g}^{-1}\right)$ than that shown for $\mathrm{Cd}\left(12 \mathrm{mg} \mathrm{g}^{-}\right.$ ${ }^{1}$ ). This fact indicates a higher affinity the adsorption sites of $\mathrm{HA}$ for $\mathrm{Pb}$. Comparatively, adsorption experiments were performed with HA Leonardite (standard IHSS) and oxidized coal. For the determination of the thermodynamic parameters, adsorption experiments were performed at three different temperatures 
$\left(15,25,35^{\circ} \mathrm{C}\right)$. The adsorption process is endothermic nature, with a value of enthalpy of $7.86 \mathrm{~kJ} \mathrm{~mol}^{-1}$ and a value of entropy $80.54 \mathrm{~mol}^{-1} \mathrm{~K}^{-1}$. The adsorption kinetics follows a kinetic model of the pseudo-second-order with rate constants $0.065 \mathrm{~g} \mathrm{mg}^{-1} \mathrm{~min}^{-1}$ and $0.025 \mathrm{mg} \mathrm{g}^{-1} \mathrm{~min}^{-1}$ for $\mathrm{Pb}\left(\mathrm{Co} 17.60 \mathrm{mg} \mathrm{L}^{-1}\right)$ and $\mathrm{Cd}(\mathrm{Co}$ $\left.6.41 \mathrm{mg} \mathrm{L}^{-1}\right)$, respectively. The results obtained in this study show that the coal HA can optionally be employed for removing heavy metals from aqueous media, but studies for their potential should however be realized.

\section{Keywords}

Extraction; Humic acid; adsorption; isotherms; thermodynamics; kinetics; heavy metals. 


\section{Sumário}

1 INTRODUÇÃO

2 OBJETIVOS E RELEVÂNCIA DO TRABALHO 20

2.1. Objetivo geral 20

2.2. Objetivos específicos $\quad 20$

3 REVISÃO BIBLIOGRÁFICA 21

3.1. Carvão mineral 21

3.1.1. Oxidação do Carvão 22

3.2. Sustâncias Húmicas 23

3.2.1. Extração de Sustâncias Húmicas 26

3.2.2. Propriedades e caracterização de ácidos húmicos 27

3.3. Poluição ambiental por metais pesados 30

3.3.1. Chumbo e Cadmio 33

3.4. Uso potencial de ácidos húmicos como adsorventes no tratamento de efluentes 35

3.5. Mecanismos de adsorção 38

3.5.1. Isotermas de adsorção $\quad 40$

3.5.2. Cinética de adsorção 45

3.5.3. Parâmetros termodinâmicos do processo de adsorção 50

4 MATERIAIS E MÉTODOS 53

4.1. Material empregado para a extração do $\mathrm{AH}$

4.2. Métodos 53

4.2.1. Preparação da amostra de Carvão Bruto 53

4.2.2. Oxidação do Carvão bruto 54

4.2.3. Extração do Ácido Húmico 54 
4.2.4. Purificação do Ácidos Húmico 56

4.3. Caracterização do AH obtido 57

4.3.1. Determinação da composição elementar 57

4.3.2. Determinação do teor de acidez do $\mathrm{AH}$

4.3.3. Espectroscopia na região do infravermelho 60

4.3.4. Análise termogravimétrica 60

4.3.5. Análise de microscopia eletrônica de varredura (MEV) 60

4.4. Ensaios de Adsorção 61

4.4.1. Preparo das soluções 61

4.4.2. Avaliação do efeito do $\mathrm{pH} \quad 61$

4.4.3. Isotermas de Adsorção 62

4.5. Determinação do modelo cinético de adsorção de $\mathrm{Pb}^{+2}$ e $\mathrm{Cd}^{+2}$ em AH 64

4.6. Determinação dos parâmetros termodinâmicos

De adsorção de $\mathrm{Pb}^{+2}$ em AH 65

5 RESULTADOS E DISCUSSÃO 66

5.1. Rendimento da extração de $\mathrm{AH} \quad 66$

5.2. Características do AH obtido 66

5.2.1. Composição elementar 66

5.2.2. Teor de acidez do AH 68

5.2.3. Grupos funcionais determinados por FTIR 69

5.2.4. Comportamento térmico do AH 72

5.2.5. Micromorfologia determinada por microscopia

eletrônica de varredura (MEV) $\quad 74$

5.3. Ensaios de adsorção 75

5.3.1. Efeito do $\mathrm{pH}$ na capacidade de adsorção 75

5.3.2. Isotermas de adsorção 77

5.4. Cinética de adsorção 90

5.5. Parâmetros termodinâmicos da adsorção de

$\begin{array}{ll}\mathrm{Pb}^{+2} \mathrm{em} \mathrm{AH} & 97\end{array}$

$\begin{array}{ll}6 \text { CONCLUSÕES } & 103\end{array}$ 


\section{Lista de figuras}

Figura 3.1 - Reservas mundiais de carvão mineral - 2008.

Figura 3.2 - Estrutura proposta para o $\mathrm{AH}$. 28

Figura 3.3 - Espectros de FTIR de AH Extraídos de Solo. 29

Figura 3.4 - Curvas TG-DTA em atmosfera de ar de três

ácidos húmicos extraídos de turfa. 30

Figura 3.5 - Distribuição das vazões de retirada para

diferentes usos consecutivos da agua.

Figura 3.6 - Sítios reativos com afinidades de ligação

de forças fracas e altamente estáveis.

Figura 3.7 - Grupos de ligação disponíveis na molécula de $\mathrm{AH}$.

Figura 3.8 -Tipos de adsorção.

Figura 3.9 - Exemplos de equilíbrio da adsorção em

sistemas ambientais naturais e artificiais.

Figura 4.1 - Fluxograma das etapas da preparação do Carvão mineral

Figura 4.2 - Fluxograma da extração do $\mathrm{AH}$

Procedimento batelada IHSS.

Figura 4.3 - Fotografia do processo de dialises

com água destilada.

Figura 4.4 - Fotografia do sistema batelada para

a determinação do teor da acidez total e carboxílica.

Figura 5.1 - Espectro de infravermelho do AH obtido

a partir de carvão mineral.

Figura 5.2 - Espectro de infravermelho do $\mathrm{AH}$ padrão da Leonardita IHSS. 
Figura 5.3 - Registro de TGA da amostra de AH. 72

Figura 5.4 - Micrografias obtidas do AH.

Figura 5.5 - Capacidade de adsorção do AH

em função do $\mathrm{pH}$ para o $\mathrm{Pb}^{+2}$.

Figura 5.6 - Capacidade de adsorção do AH

em função do $\mathrm{pH}$ para o $\mathrm{Cd}^{+2}$. $\quad 76$

Figura 5.7 - Isoterma de adsorção para o $\mathrm{Pb}^{+2}$. 77

Figura 5.8 - Isoterma de adsorção para o $\mathrm{Cd}^{+2}$. 78

Figura 5.9 - Linearização da isoterma de Langmuir - $\mathrm{Pb}^{+2}$. 79

Figura 5.10 - Linearização da isoterma de Freundlich - $\mathrm{Pb}^{+2}$. $\quad 79$

Figura 5.11 - Linearização da isoterma de Redlich-Peterson - $\mathrm{Pb}^{+2} . \quad 80$

Figura 5.12 - Linearização da isoterma de Langmuir - $\mathrm{Cd}^{+2}$. 80

Figura 5.13 - Linearização da isoterma de Freundlich - $\mathrm{Cd}^{+2}$. 81

Figura 5.14 - Linearização da isoterma de Redlich-Peterson - $\mathrm{Cd}^{+2} . \quad 81$

Figura 5.15 - Comparação de isotermas de adsorção - $\mathrm{Pb}^{+2}$. 84

Figura 5.16 - Comparação de isotermas de adsorção - $\mathrm{Cd}^{+2}$. 85

Figura 5.17 - Mapeamento (a) e espectro (b) por EDS

da superfície do $\mathrm{AH}$ após adsorção de $\mathrm{Pb}^{+2}$.

Figura 5.18 - Mapeamento (a) e espectro (b) por EDS

da superfície do $\mathrm{AH}$ após adsorção de $\mathrm{Cd}^{+2}$.

Figura 5.19 - Espectro FTRI do AH na ausência e

na presença de $\mathrm{Pb}+2(100 \mathrm{mg} / \mathrm{l})$ e $\mathrm{Cd}+2(40 \mathrm{mg} / \mathrm{l})$. 88

Figura 5.20 - Cinética da adsorção de $\mathrm{Pb}+2$. 90

Figura 5.21 - Cinética da adsorção de $\mathrm{Cd}+2$. 91

Figura 5.22 - Representação gráfica do modelo

de Langergren (pseudo primeira ordem) para o de $\mathrm{Pb}^{+2}$. 92

Figura 5.23 - Representação gráfica do modelo de

pseudo segunda ordem para o $\mathrm{Pb}^{+2}$. 92

Figura 5.24 - Representação gráfica do modelo de

Langergren (pseudo primeira ordem) para o $\mathrm{Cd}^{+2}$. 93

Figura 5.25 - Representação gráfica do modelo de

pseudo segunda ordem para o $\mathrm{Cd}^{+2}$. 94

Figura 5.26 - Cinética da adsorção do $\mathrm{Pb}^{+2}$. 95 
Figura 5.27- Representação gráfica do modelo de pseudo segunda ordem para o $\mathrm{Pb}^{+2}$.

Figura 5.28 - Representação da equação de

Arrhenius para o sistema $\mathrm{AH}-\mathrm{Pb}$.

Figura 5.29 - Representação do efeito da variação

da temperatura na isoterma de $\mathrm{Pb}^{+2}$.

Figura 5.30 - Determinação dos parâmetros

termodinâmicos empregando Kads de Langmuir.

Figura 5 31: Determinação dos parâmetros

termodinâmicos empregando a constante aparente.

102 


\section{Lista de tabelas}

Tabela 3.1 - Questões ambientais que envolvem SH's

Tabela 3.2 - Setores industriais com maior presença

de íons de metais pesados nos efluentes.

Tabela 3.3 - Limites máximos permissíveis para

o lançamento de efluentes industrias.

Tabela 3.4 - Comparação entre fisissorção e quimissorção.

Tabela 4.1- Características e Composição Elementar

Tabela 4.2 - Padrões empregados nas curvas de calibração de AA.

Tabela 4.3 - Condições empregadas na avaliação

do $\mathrm{pH}$ nos ensaios de adsorção

Tabela 4-4 - Condições empregadas nas Isotermas

de Adsorção dos cátions de $\mathrm{Pb}^{+2} \mathrm{e} \mathrm{Cd}^{+2}$.

Tabela 4.5 - Condições empregadas na adsorção

com AH (IHSS) e carvão bruto oxidado.

Tabela 4.6 - Condições empregadas nos ensaios

cinéticos para a adsorção dos cátions de $\mathrm{Pb}^{+2} \mathrm{e} \mathrm{Cd}^{+2}$.

Tabela 5.1 - Composição Elementar e razões atômicas

do AH de carvão mineral

Tabela 5.2 - Tipos de acidez determinada no AH

de carvão mineral.

Tabela 5.3 - Principais bandas de adsorção na

região do IV observadas nos espectros $\mathrm{AH}$

Tabela 5.4 - Resultado da Análise termogravimétrica.

Tabela 5.5 - Composição elementar do AH obtido pelo método EDS-MEV. 
Tabela 5.6 - Mudanças do pH da solução após o processo de adsorção.

Tabela 5.7 - Parâmetros dos modelos de Langmuir, Freundlich, e Redlich-Peterson para o $\mathrm{Pb}^{+2}$ e $\mathrm{Cd}^{+2} \mathrm{em} \mathrm{AH}$.

Tabela 5.8 - Constantes de adsorção de acordo com a equação de Langmuir para a adsorção de $\mathrm{Pb}^{+2}$ em três diferentes adsorventes.

Tabela 5.9 - Parâmetros de adsorção de acordo com a equação de Langmuir para a adsorção de $\mathrm{Cd}^{+2} \mathrm{em}$ AH de carvão mineral e Leonardita.

Tabela 5.10 - Comparação de capacidade de adsorção $\left(\mathrm{mg} \mathrm{g}^{-1}\right)$ de diferentes adsorventes para adsorção de $\mathrm{Pb}$ e $\mathrm{Cd}$.

Tabela 5.11 - Variação da intensidade relativa do AH após adsorção de Chumbo e Cadmio.

Tabela 5.12 - Parâmetros cinéticos segundo o modelo de pseudo primeira e pseudo segunda ordem para adsorção de $\mathrm{Pb}+2 \mathrm{em} \mathrm{AH}$.

Tabela 5.13 - Parâmetros cinéticos segundo o modelo de pseudo primeira e de pseudo segunda ordem para $\circ \mathrm{Cd}^{+2}$.

Tabela 5.14 - Parâmetros cinéticos de pseudo segundo ordem em função da temperatura para a adsorção de $\mathrm{Pb}^{+2} \mathrm{em} \mathrm{AH}$.

Tabela 5.15 - Determinação da variação da capacidade de adsorção e da constante de Langmuir com a temperatura para o $\mathrm{Pb}^{+2}$.

Tabela 5.16 - Parâmetros termodinâmicos para a remoção de $\mathrm{Pb}^{+2}$ determinados utilizando o parâmetro $\mathrm{K}_{\text {ads. }}$.

Tabela 5.17 - Determinação da variação da constante de equilíbrio aparente e da energia padrão de Gibbs com a temperatura.

Tabela 5.18 - Parâmetros termodinâmicos da adsorção de $\mathrm{Pb}+2$ em $\mathrm{AH}$ a pH 5 determinados utilizando $\mathrm{Kd}$. 


\section{Lista de símbolos}

\section{Caracteres latinos:}

AA absorção atômica

AF ácido fúlvico

AH ácido húmico.

Acarboxilica acidez carboxílica, mmol carga $\mathrm{g}^{-1}$.

Afenólica acidez fenólica, mmol carga $\mathrm{g}^{-1}$.

Atotal acidez total, mmol carga $\mathrm{g}^{-1}$.

Ao constante de Arrhenius, $\mathrm{g} \mathrm{min}^{-1} \mathrm{mg}^{-1}$.

Ce concentração do cátion remanescente na solução, $\mathrm{mg} \mathrm{L}^{-1}$.

h taxa inicial de adsorção, $\mathrm{mg} \mathrm{g}^{-1} \mathrm{~min}^{-1}$.

IHSS Sociedade Internacional de Substâncias Húmicas

Kads constante de Langmuir, relacionada à energia de adsorção, $\mathrm{L} \mathrm{mg}^{-1}$

$K_{\text {eq }}$ constante de equilíbrio condicional de adsorção.

$\mathbf{K}_{\mathbf{f}}$ constante de Freundlich, relativa à capacidade de adsorção, $\mathrm{L} \mathrm{g}^{-1}$.

$K_{\text {RP }}$ constante de Redlich-Peterson, relativa à capacidade de adsorção,

k1 taxa de adsorção de pseudo-primeira ordem, $\min ^{-1}$.

k2 taxa de adsorção de pseudo-segunda ordem, $\mathrm{g} \mathrm{mg}^{-1} \mathrm{~min}^{-1}$.

$q_{e} \quad$ capacidade de adsorção no equilíbrio $\mathrm{mg} \mathrm{g}^{-1}$.

$q_{\max }$ capacidade de adsorção máxima do o modelo de Langmuir, $\mathrm{mg} \mathrm{g}^{-1}$.

qt capacidade de adsorção no tempo $\mathrm{t}, \mathrm{mg} \mathrm{g}^{-1}$.

$\mathbf{R}$ constante dos gases $\left(8,314 \mathrm{~J} \mathrm{~mol}^{-1} \mathrm{~K}^{-1}\right)$

SH sustâncias húmicas.

T temperatura em $\mathrm{K}$.

1/n constante de Freundlich, representa a intensidade de adsorção (adimensional). 


\section{Caracteres gregos:}

iL atividade do componente i, na fase líquida.

ir atividade do componente $\mathrm{i}$, na interfase.

$\mathbf{G}^{\circ}$ energia padrão de Gibbs, $\mathrm{kJ} \mathrm{mol}^{-1}$.

$\mathbf{H}^{\circ}$ é a variação de entalpia padrão, $\mathrm{kJ} \mathrm{mol}^{-1}$.

$\mathbf{S}^{\circ} \quad$ é a variação de entropia padrão, $\mathrm{J} \mathrm{mol} \mathrm{K}^{-1}$.

$\boldsymbol{\mu} \mathbf{0}_{\mathbf{i}}$ potencial químico padrão do componente i, na interfase.

$\boldsymbol{\mu}^{\mathrm{LO}_{\mathrm{i}}} \quad$ potencial químico padrão do componente $\mathrm{i}$, na fase líquida.

$\boldsymbol{\theta}$ fração relativa a quantidade de sítios ativos do $\mathrm{AH}$ ocupados. 